\title{
Dimension of countable intersections of some sets arising in expansions in non-integer bases
}

\author{
by \\ David Färm (Lund), Tomas Persson (Warszawa) \\ and Jörg Schmeling (Lund)
}

\begin{abstract}
We consider expansions of real numbers in non-integer bases. These expansions are generated by $\beta$-shifts. We prove that some sets arising in metric number theory have the countable intersection property. This allows us to consider sets of reals that have common properties in a countable number of different (non-integer) bases. Some of the results are new even for integer bases.
\end{abstract}

1. Introduction. Metric number theory is a classical and important field in mathematics. Nowadays its applications go far beyond the study of diophantine equations and are central in KAM-theory or linearisation of parabolic fixed points in complex dynamics.

We will address the question of the sizes of some sets defined by their properties of digits in a given base. The main purpose is not to restrict to integer bases but to allow real bases arising from $\beta$-shifts. The main difficulty in this set-up is that unlike integer bases most real bases have languages that do not have the specification property. In particular the associated symbolic dynamics are not of finite type. Hence the natural net-measure defined by cylinders will not satisfy the regularity conditions needed for the known methods to check the large intersection properties. Our main method is to use the (fast) approximation of a general $\beta$-shift by $\beta$-shifts of finite type and not to verify the large intersection property directly in the general $\beta$-shift.

The first class of sets we are interested in are sets of points whose $\omega$-limit set under the associate dynamics avoids a given point. If one chooses the avoided point to be the origin this set consists of the points which are worst approximable by "rationals" of the form $p / \beta^{i}, i \in \mathbb{N}$. Such sets are known as the sets of badly approximable numbers and play a central role in classical diophantine approximation. For a fixed base $\beta$ these sets were studied in

2010 Mathematics Subject Classification: Primary 37E05; Secondary 37C45, 11J83.

Key words and phrases: beta-shift, Hausdorff dimension, non-typical points. 
detail by Nilsson [8, [9]. One of the authors, Färm, proved that these sets have the large intersection property provided $\beta$ gives rise to a subshift of finite type (see [3]). Our purpose is to avoid this condition. More precisely we prove that for a given sequence of bases $\left(\beta_{n}\right)_{n}$, the Hausdorff dimension of the set of points whose orbit in any of the $\beta$-shifts $f_{\beta_{n}}:[0,1] \rightarrow[0,1)$, $f_{\beta_{n}}(x)=\beta_{n} x(\bmod 1)$ avoids the given point (jointly for all bases) is 1 . To prove this we will use a modification of Schmidt's $(\alpha, \beta)$-game. (In this paper we will call it the $(\alpha, \gamma)$-game, since $\beta$ will be used for $\beta$-shifts.) Another related question is about sets having a given rate of approximation. This question (for fixed $\beta$ ) was addressed in [11].

The second class of sets we are interested in are sets of reals having the property that the frequencies of occurrence of digits or finite words do not exist. This is a subset of the irregular set studied in [2]. Färm proved that such sets have full dimension and are in Falconer's intersection class provided $\beta$ gives rise to a subshift of finite type (see [4]). We will prove that for any given $\beta$ these sets are in Falconer's intersection class and hence have the large intersection property. This implies that for a given sequence $\beta_{n}$ the set of reals having no converging frequencies of digits (words) in any of the bases $\beta_{n}$ has full dimension. The sets in question are defined by "semi-regular" points, i.e. points that have a given Bernoulli (Markov) measure as an accumulation point of their empirical measures. In this weak sense it provides a contrast to the well-known fact (Schmidt, Feldman and Smorodinsky, and others) that for a given Bernoulli measure in (integer) base $g_{1}$, a.e. point has equal frequency of all digits in any logarithmically independent base $g_{2}$.

We also want to mention that the main results of the paper are of a more general nature than the applications require. Therefore the techniques might be applied to other situations as well.

\section{Notation and theory}

2.1. $\beta$-shifts and $\beta$-expansions. Let $[x]$ denote the integer part of the real number $x$, and let $\lfloor x\rfloor$ denote the largest integer strictly smaller than $x$. Let $\beta>1$. To any $x \in[0,1]$ we associate the sequence $d(x, \beta)=$ $\left(d_{n}(x, \beta)\right)_{n=0}^{\infty} \in\{0,1, \ldots,\lfloor\beta\rfloor\}^{\mathbb{N}}$ defined by

$$
d_{n}(x, \beta):=\left[\beta f_{\beta}^{n}(x)\right],
$$

where $f_{\beta}(x)=\beta x(\bmod 1)$. The closure of the set

$$
\{d(x, \beta): x \in[0,1)\}
$$

is denoted by $S_{\beta}$ and called the $\beta$-shift. We will denote the set of all allowed finite words in $S_{\beta}$ by $S_{\beta}^{*}$. The sets $S_{\beta}$ and $S_{\beta}^{*}$ are invariant under the leftshift $\sigma:\left(i_{n}\right)_{n=0}^{\infty} \mapsto\left(i_{n+1}\right)_{n=0}^{\infty}$ and the map $d(\cdot, \beta): x \mapsto d(x, \beta)$ satisfies the equality $\sigma^{n}(d(x, \beta))=d\left(f_{\beta}^{n}(x), \beta\right)$. If we order $S_{\beta}$ with the lexicographical 
ordering then the map $d(\cdot, \beta)$ is one-to-one and increasing. The subshift $S_{\beta}$ satisfies

$$
S_{\beta}=\left\{\left(j_{k}\right)_{k=0}^{\infty}: \sigma^{n}\left(j_{k}\right)_{k=0}^{\infty}<d(1, \beta) \forall n\right\} .
$$

Parry proved in $[10$, that the map $\beta \mapsto d(1, \beta)$ is strictly increasing. For a sequence $\left(j_{k}\right)_{k=0}^{\infty}$ there is a $\beta>1$ such that $\left(j_{k}\right)_{k=0}^{\infty}=d(1, \beta)$ if and only if $\sigma^{n}\left(\left(j_{k}\right)\right)<\left(j_{k}\right)$ for every $n>0$. The number $\beta$ is then the unique positive solution of the equation

$$
1=\sum_{k=0}^{\infty} \frac{d_{k}(1, \beta)}{x^{k+1}} .
$$

One observes that the fact that the map $\beta \mapsto d(1, \beta)$ is strictly increasing together with (2.1) implies that $S_{\beta_{1}} \subseteq S_{\beta_{2}}$ if and only if $\beta_{1} \leq \beta_{2}$.

If $x \in[0,1]$ then

$$
x=\sum_{k=0}^{\infty} \frac{d_{k}(x, \beta)}{\beta^{k+1}} .
$$

This formula can be seen as an expansion of $x$ in the non-integer base $\beta$, and thereby generalises the ordinary expansion in integer bases.

We let $\pi_{\beta}: S_{\beta} \rightarrow[0,1)$ be defined by

$$
\pi_{\beta}:\left(i_{k}\right)_{k=0}^{\infty} \mapsto \sum_{k=0}^{\infty} \frac{i_{k}}{\beta^{k+1}} .
$$

Hence, $\pi_{\beta}(d(x, \beta))=x$ for any $x \in[0,1)$ and $\beta>1$.

A cylinder $s$ is a subset of $S_{\beta}$ such that

$$
s=\left\{\left(j_{k}\right)_{k=0}^{\infty} \in S_{\beta}: i_{k}=j_{k}, 0 \leq k<n\right\}
$$

for some $n$ and some sequence $\left(i_{k}\right)_{k=0}^{\infty}$. We then say that $s$ is an $n$-cylinder or a cylinder of generation $n$ and write

$$
s=\left[i_{0} \cdots i_{n-1}\right] .
$$

The set $\pi_{\beta}(s)$ is a half-open interval. We will also call $\pi_{\beta}(s)$ a cylinder, and sometimes identify the cylinders $s$ and $\pi_{\beta}(s)$.

We emphasise that if $s=\left[i_{0} \cdots i_{n-1}\right]$ is a non-empty $n$-cylinder, then there is a largest $k \geq 0$ such that

$$
s=[i_{0} \cdots i_{n-1} \underbrace{0 \cdots 0}_{k \text { zeros }}] .
$$

The set

$$
s 0=[i_{0} \cdots i_{n-1} \underbrace{0 \cdots 0}_{k+1 \text { zeros }}]
$$

then satisfies $\sigma^{n+k+1}(s 0)=S_{\beta}$, so $\pi_{\beta}(s 0)$ is just a scaling of $[0,1)$ by a factor $1 / \beta^{n+k+1}$. 
In the forthcoming we will restrict to the case $\beta \in(1,2]$ in order to keep the notations and proofs as simple as possible. The general case is just a technical/notational adjustment and does not require any new ideas.

2.2. The $(\alpha, \gamma)$-game. We will use a one-dimensional version of a settheoretic game that was introduced by W. Schmidt in [12]. In our case, the game is played on the unit interval $[0,1]$ equipped with Euclidean metric. There are two players, Black and White, and two fixed numbers $\alpha, \gamma \in(0,1)$. A set $E \subset[0,1]$ is given before the game starts. The game is then played according to the following rules.

In the initial step Black chooses any closed interval $B_{0}$, and then White chooses a closed interval $W_{0} \subset B_{0}$ such that $\left|W_{0}\right|=\alpha\left|B_{0}\right|$.

Then the following step is repeated. At step $k$ Black chooses a closed interval $B_{k} \subset W_{k-1}$ such that $\left|B_{k}\right|=\gamma\left|W_{k-1}\right|$. Then White chooses a closed interval $W_{k} \subset B_{k}$ such that $\left|W_{k}\right|=\alpha\left|B_{k}\right|$.

It is clear that the set

$$
\bigcap_{k=0}^{\infty} W_{k}=\bigcap_{k=0}^{\infty} B_{k}
$$

will always consist of exactly one point. A set $E$ is said to be $(\alpha, \gamma)$-winning if White can always achieve that

$$
\bigcap_{k=0}^{\infty} W_{k} \subset E .
$$

A set $E$ is said to be $\alpha$-winning if it is $(\alpha, \gamma)$-winning for all $\gamma$.

For us, the key property of $\alpha$-winning sets proved by Schmidt [12] can be summarised as follows.

Proposition 1. If the set $E \subset[0,1]$ is $\alpha$-winning for some $\alpha>0$, then $\operatorname{dim}_{H}(E)=1$.

Proposition 2. Let $\alpha>0$ and let $\left(E_{i}\right)_{i=1}^{\infty}$ be a sequence of $\alpha$-winning sets. Then the set $\bigcap_{i=1}^{\infty} E_{i}$ is also $\alpha$-winning.

2.3. A modified $(\alpha, \gamma)$-game. We will use the following modification of the $(\alpha, \gamma)$-game. Let $\alpha_{0}, \gamma_{0} \in(0,1)$ be fixed.

In the initial step Black chooses any closed interval $B_{0}$, and then White chooses an $\alpha \in\left[\alpha_{0}, 1\right]$ and a closed interval $W_{0} \subset B_{0}$ such that $\left|W_{0}\right|=\alpha\left|B_{0}\right|$.

Then the following step is repeated. At step $k$ Black chooses $\gamma \in\left[\gamma_{0}, 1\right]$ and a closed interval $B_{k} \subset W_{k-1}$ such that $\left|B_{k}\right|=\gamma\left|W_{k-1}\right|$. Then White chooses a new $\alpha \in\left[\alpha_{0}, 1\right]$ and a closed interval $W_{k} \subset B_{k}$ such that $\left|W_{k}\right|=\alpha\left|B_{k}\right|$. 
We say that a set $E$ is $\left(\alpha_{0}, \gamma_{0}\right)$-winning if White can always achieve

$$
\bigcap_{k=0}^{\infty} W_{k} \subset E,
$$

and $\alpha_{0}$-winning if this holds for all $\gamma_{0}$. We start by noting the connection to the original game.

Proposition 3. If the set $E \subset[0,1]$ is $\alpha_{0}$-winning in the modified game, then $E$ is also $\alpha_{0}$-winning in the original $(\alpha, \gamma)$-game.

If the set $E \subset[0,1]$ is $\alpha_{0}$-winning in the modified game, then it is also winning for all smaller $\alpha_{0}$.

Proof. Let us agree to sometimes call White she and Black he. We emphasise however that the gender of the players has no influence on the proof.

We prove the first statement. Assume $E$ is $\alpha_{0}$-winning in the modified game. So White always wins no matter what $\gamma_{k}$ Black chooses. In particular if Black plays as below White still wins. Assume that Black chooses his $\gamma$ as follows.

In the initial step Black chooses any closed interval $B_{0}$, and then White chooses an $\alpha(0) \in\left[\alpha_{0}, 1\right]$ and a closed interval $W_{0} \subset B_{0}$ such that $\left|W_{0}\right|=\alpha(0)\left|B_{0}\right|$.

Then the following step is repeated. At step $k$ Black chooses $\gamma(k)=$ $\gamma_{0} \frac{\alpha_{0}}{\alpha(k-1)}$ and a closed interval $B_{k} \subset W_{k-1}$ such that $\left|B_{k}\right|=\gamma(k)\left|W_{k-1}\right|$. Then White chooses a new $\alpha(k) \in\left[\alpha_{0}, 1\right]$ and a closed interval $W_{k} \subset$ $B_{k}$ such that $\left|W_{k}\right|=\alpha(k)\left|B_{k}\right|$.

Since $\gamma(k) \geq \gamma_{0} \alpha_{0}$ for all $k$, we know by assumption that White can make sure that $\bigcap_{k=0}^{\infty} W_{k} \subset E$ for all $\gamma_{0}$. Note that now we have $\left|B_{k+1}\right|=\alpha_{0} \gamma_{0}\left|B_{k}\right|$ and $|W(k)| \geq \alpha_{0}|B(k)|$ for all $k$. We can interpret this as the original $(\alpha, \gamma)$ game where $\alpha=\alpha_{0}$ and $\gamma=\gamma_{0}$ in which White uses a strategy where she sometimes does not use all her freedom given by $\alpha$ and lets Black use this freedom instead. Thus, $E$ is $\alpha_{0}$-winning in the original $(\alpha, \gamma)$-game.

The second statement of the proposition is obviously true.

COROLlary 1 . If the set $E \subset[0,1]$ is $\alpha$-winning in the modified game for some $\alpha>0$, then $\operatorname{dim}_{H}(E)=1$.

Proof. This follows immediately from Propositions 1 and 3

From now on we will only use the modified game in the rest of the paper.

The proof of the following proposition is a slight modification of the proof of Theorem 2 from 12 .

Proposition 4. Let $\alpha_{0}>0$ and let $\left(E_{i}\right)_{i=1}^{\infty}$ be a sequence of $\alpha_{0}$-winning sets. Then the set $\bigcap_{i=1}^{\infty} E_{i}$ is also $\alpha_{0}$-winning. 
Proof. Let $\alpha_{0}>0$ and let $\left(E_{i}\right)_{i=1}^{\infty}$ be a sequence of $\alpha_{0}$-winning sets. We will produce a strategy for White that ensures that $\bigcap_{k=0}^{\infty} W_{k} \subset \bigcap_{i=1}^{\infty} E_{i}$.

Given $\gamma_{0}$, at the first, third, fifth,... turn, White plays according to a strategy from the $\left(\alpha_{0}, \alpha_{0} \gamma_{0}^{2}\right)$-game which ensures that $\bigcap_{k=0}^{\infty} W_{k} \subset E_{1}$. This strategy will be completely independent of what White does in the rest of her turns.

At the second, sixth, tenth,... turn, White plays according to a strategy from the $\left(\alpha_{0},\left(\alpha_{0} \gamma_{0}\right)^{3} \gamma_{0}\right)$-game which ensures that $\bigcap_{k=0}^{\infty} W_{k} \subset E_{2}$. This strategy will be completely independent of what White does in the rest of her turns.

In general, at play number $k$, where $k=2^{l-1} \bmod 2^{l}$, White plays as if she was playing the $\left(\alpha_{0},\left(\alpha_{0} \gamma_{0}\right)^{2^{l}-1} \gamma_{0}\right)$-game ensuring that $\bigcap_{k=0}^{\infty} W_{k} \subset E_{l}$.

Thus, playing according to this strategy, White can make sure that $\bigcap_{k=0}^{\infty} W_{k} \subset \bigcap_{i=1}^{\infty} E_{i}$.

2.4. Falconer's intersection classes. In [6] Falconer characterises a family of classes $\mathcal{G}^{s}$ of sets with the property that any countable intersection of sets in $\mathcal{G}^{s}$ is in $\mathcal{G}^{s}$ and the Hausdorff dimension of any set in $\mathcal{G}^{s}$ is at least $s$. Below we give one of the many possible definitions (see also [5, 7]) of the classes $\mathcal{G}^{s}$ and some results about them. We will only formulate this for subsets of the unit interval, although the construction in [6] is done in $\mathbb{R}^{n}$.

We denote by $|A|$ the diameter of a set $A$ and define

$$
\mathcal{M}_{\infty}^{s}(F)=\inf \left\{\sum_{i}\left|U_{i}\right|^{s}: F \subset \bigcup_{i} U_{i}\right\} .
$$

where each $U_{i}$ is of the form $\left[a / 2^{i},(a+1) / 2^{i}\right), a \in\left\{0,1, \ldots, 2^{i}-1\right\}$. Note that that $\mathcal{M}_{\infty}^{s}(F) \leq|F|^{s}$ for any set $F$. We say that a set $E \subset[0,1]$ is in the class $\mathcal{G}^{s}$ if $E$ is a $G_{\delta}$ set and $\mathcal{M}_{\infty}^{s}(E \cap I)=|I|^{s}$ for any interval $I \subset[0,1]$.

We will use the following properties of the classes $\mathcal{G}^{s}$. For proofs, see [6].

Proposition 5. A $G_{\delta}$ set $E$ is in the class $\mathcal{G}^{s}$ if and only if there is a constant $C>0$ such that $\mathcal{M}_{\infty}^{s}(E \cap I)>C|I|^{s}$ for any interval $I \subset[0,1)$.

Proposition 6. If $E$ is in $\mathcal{G}^{s}$, then $\operatorname{dim}_{\mathrm{H}}(E) \geq s$. If $E_{k}, k \in \mathbb{N}$, are sets in $\mathcal{G}^{s}$, then $\bigcap_{k \in \mathbb{N}} E_{k}$ is in $\mathcal{G}^{s}$.

Proposition 7. $\mathcal{G}^{s}=\bigcap_{t<s} \mathcal{G}^{t}$.

3. Results. The main results of this paper are the following two theorems. The theorems aim towards the applications in Section 4 , but can be used for a wider set of purposes. Both theorems are approximation theorems in the following sense. If we approximate a subset of $S_{\beta}$ by subsets of $S_{\beta_{n}}$, where $S_{\beta_{n}}$ is of finite type, and prove that all approximations are either $\alpha_{0}$-winning or in the class $\mathcal{G}^{s}$, can we then conclude that the subset of $S_{\beta}$ is either $\alpha_{0}$-winning or in the class $\mathcal{G}^{s}$ ? 
Theorem 1. Let $\beta \in(1,2)$ and let $\left(\beta_{n}\right)_{n=1}^{\infty}$ be any sequence with $\beta_{n} \in$ $(1, \beta)$ for all $n$ such that $\beta_{n} \rightarrow \beta$ as $n \rightarrow \infty$. Let also $E \subset S_{\beta}$ and $\alpha \in(0,1)$. If $\pi_{\beta_{n}}\left(E \cap S_{\beta_{n}}\right)$ is $\alpha_{0}$-winning for $\alpha_{0}=\alpha$ for all $n$, then $\pi_{\beta}(E)$ is $\alpha_{0}$-winning for any $\alpha_{0} \leq \min \{1 / 16, \alpha / 4\}$.

Theorem 2. Let $\beta \in(1.541,2)$ and let $\left(\beta_{n}\right)_{n=1}^{\infty}$ be any sequence with $\beta_{n} \in(1.541, \beta)$ for all $n$ such that $\beta_{n} \rightarrow \beta$ as $n \rightarrow \infty$. Assume that $E \subset S_{\beta}$ and $\pi_{\beta_{n}}\left(E \cap S_{\beta_{n}}\right)$ is in $\mathcal{G}^{s}$ for all $n$. If $F$ is a $G_{\delta}$ set such that $F \supset \pi_{\beta}(E)$, then $F$ is also in $\mathcal{G}^{s}$.

REMARK. The constant 1.541 is a technical condition for our proof of Lemma 1. There seems to be no general reason why the statement should fail for smaller $\beta$. The constant is the inverse of the transversality constant appearing in $[13$.

\section{Applications of Theorems 1 and 2}

4.1. The $(\alpha, \gamma)$-game and $\beta$-shifts. Consider the set

$$
G_{\beta}(x):=\left\{y \in[0,1): x \notin \overline{\bigcup_{n=0}^{\infty} f_{\beta}^{n}(y)}\right\}
$$

which is the set of all points for which the forward orbit under the map $f_{\beta}$ is bounded away from some given point $x \in[0,1]$. In $S_{\beta}$ this set is represented by

$$
E_{\beta}(x)=\bigcup_{n=1}^{\infty}\left\{\left(y_{i}\right)_{i=0}^{\infty} \in S_{\beta}: x_{0} \ldots x_{n-1} \neq y_{k} \ldots y_{k+n-1} \forall k \geq 0\right\},
$$

where $\pi_{\beta}\left(\left(x_{i}\right)_{i=0}^{\infty}\right)=x$.

Consider $\beta$ such that the expansion of 1 terminates, i.e.

$$
d(1, \beta)=j_{0} \ldots j_{k-1} 0^{\infty} .
$$

The set of such $\beta$ is dense in $(1,2)$. Thus, given $\beta \in(1,2)$ we can find a sequence $\left(\beta_{n}\right)_{n=1}^{\infty}$ converging to $\beta$ from below such that the expansion of 1 terminates for each $\beta_{n}$. Let $x=\pi_{\beta}\left(\left(x_{i}\right)_{i=0}^{\infty}\right)$, where $\left(x_{i}\right)_{i=0}^{\infty} \in S_{\beta}$. If $\left(x_{i}\right)_{i=0}^{\infty} \notin S_{\beta_{n}}$, we have

$$
\pi_{\beta_{n}}\left(E_{\beta}(x) \cap S_{\beta_{n}}\right)=[0,1)
$$

which is of course $\alpha$-winning in [0,1]. If instead $\left(x_{i}\right)_{i=0}^{\infty} \in S_{\beta_{n}}$, then

$$
\pi_{\beta_{n}}\left(E_{\beta}(x) \cap S_{\beta_{n}}\right)=G_{\beta_{n}}\left(\pi_{\beta_{n}}\left((x)_{i=0}^{\infty}\right)\right) .
$$

We obtain from [3, Theorem 1.5 and Remark 3.2] the following 
Theorem 3. Let $\beta \in(1,2)$ be such that the expansion of 1 terminates. Then for any $x \in[0,1]$ the set

$$
G_{\beta}(x)=\left\{y \in[0,1): x \notin \overline{\bigcup_{n=0}^{\infty} f^{n}(y)}\right\},
$$

is $\alpha$-winning in the modified $(\alpha, \gamma)$-game in $[0,1]$ for any $\alpha \leq 1 / 4$.

Thus $\pi_{\beta_{n}}\left(E_{\beta}(x) \cap S_{\beta_{n}}\right)$ is $\alpha$-winning for all $n$ for any $\alpha \leq 1 / 4$. Since

Theorems 1 and 3 imply

$$
G_{\beta}(x)=\pi_{\beta}\left(E_{\beta}(x)\right),
$$

Theorem 4. For any $\beta \in(1,2)$ and $x \in[0,1]$ the set

$$
G_{\beta}(x)=\left\{y \in[0,1): x \notin \overline{\bigcup_{n=1}^{\infty} f^{n}(y)}\right\},
$$

is $\alpha$-winning in the modified $(\alpha, \gamma)$-game in $[0,1]$ for any $\alpha \leq 1 / 16$.

By Proposition 4 , the intersection of $\alpha$-winning sets is $\alpha$-winning, and by Corollary 1, $\alpha$-winning sets have full dimension, so we get

Corollary 2. Let $\left(\beta_{i}\right)_{i=1}^{\infty}$ be a sequence in $(1,2)$ and let $\left(x_{i}\right)_{i=1}^{\infty}$ be a sequence of points in $[0,1]$. Then

$$
\operatorname{dim}_{\mathrm{H}}\left(\bigcap_{i=1}^{\infty} G_{\beta_{i}}\left(x_{i}\right)\right)=1 .
$$

REMARK. If we choose $\left(x_{i}\right)_{i=1}^{\infty}=0^{\infty}$ we are in the case of badly approximable numbers.

4.2. Falconer's intersection classes and $\beta$-shifts. Let $\beta \in(1.541,2)$ and let $\left(w_{j}\right)_{j=1}^{2^{m}}$ be all words of length $m$ in the alphabet $\{0,1\}$. We define

$$
\tau_{w_{j}}^{\beta}(x, n)=\#\left\{i \in\{0, \ldots, n-m-1\}: x_{i}, \ldots, x_{i+m-1}=w_{j}\right\} .
$$

For $\bar{p}=\left(p_{w_{j}}\right)_{j=1}^{2^{m}}$, we define

$$
G_{\bar{p}}^{\beta, m}=\left\{x: \frac{\tau_{w_{j}}^{\beta}(x, n)}{n-m} \rightarrow p_{w_{j}} \text { as } n \rightarrow \infty, j=1, \ldots, 2^{m}\right\}
$$

where $0 \leq p_{w_{j}} \leq 1$ can be interpreted as the frequency of the word $w_{j}$ in $d(x, \beta)$. We also define

$$
G_{\bar{p}}^{\beta, m}(n, \varepsilon)=\left\{x \in[0,1): p_{w_{j}}-\varepsilon<\frac{\tau_{w_{j}}^{\beta}(x, n)}{n-m}<p_{w_{j}}+\varepsilon, j=1, \ldots, 2^{m}\right\}
$$

and note that

$$
G_{\bar{p}}^{\beta, m} \subset \bigcap_{N=1}^{\infty} \bigcup_{n=N}^{\infty} G_{\bar{p}}^{\beta, m}(n, \varepsilon)
$$

for all $\varepsilon>0$. In 4 the following was proved: 
Proposition 8. Let $\beta \in(1,2)$ be such that the expansion of 1 terminates and $m \in \mathbb{N}$. If $\bar{p}$ is such that there is a word $v \neq 0^{m}$ with $p_{v}>0$ for $0<\varepsilon<p_{v}$, the set

$$
\bigcap_{N=1}^{\infty} \bigcup_{n=N}^{\infty} G_{\bar{p}}^{\beta, m}(n, \varepsilon)
$$

is in the class $\mathcal{G}^{s}$ for each $s \leq \operatorname{dim}_{\mathrm{H}}\left(G_{\bar{p}}^{\beta, m}\right)$.

We will use Theorem 2 to prove the following.

Theorem 5. Let $\beta \in(1.541,2)$. Then for any word $w \in S_{\beta}^{*}$ of any length $m$ the set of numbers $x \in[0,1)$ for which $\tau_{w}^{\beta}(x, n) /(n-m)$ does not converge as $n \rightarrow \infty$, in the expansion in base $\beta$, contains a set from the class $\mathcal{G}^{s}$ for each $s<1$.

Theorem 5 together with Proposition 6 leads to the following corollary.

Corollary 3. Let $\left(\beta_{i}\right)_{i=1}^{\infty}$ be any sequence in $(1.541,2)$. Then the set of numbers for which $\tau_{w}^{\beta_{i}}(x, n) /(n-m)$ does not converge as $n \rightarrow \infty$ for any word $w \in S_{\beta_{i}}^{*}$ of any length $m$, in the expansion to any base $\beta_{i}$, has Hausdorff dimension 1 .

Corollary 3 generalises Theorem 1.7 in [4], where the statement of the corollary was proved in the case where all $S_{\beta_{i}}$ are of finite type. The condition $\beta>1.541$ was however not needed in [4].

Note that since there is an ergodic measure equivalent to Lebesgue measure, the set of points for which the frequencies converge for all $\beta_{i}$ has full Lebesgue measure. It is however often the case that the exceptional points have full Hausdorff dimension. For instance, it was proved in [2] for repellers and subshifts of finite type that for finitely many Hölder continuous functions, the set of points for which the ergodic averages of the functions do not converge, has full dimension. By letting these functions be indicator functions of cylinders corresponding to some words, this implies that the set of points with no frequencies of these words has full dimension for a fixed system. Our result is stronger in the sense that we consider all (countably many) words, and countably many systems at the same time.

We will need the following lemma.

Lemma 1. Let $\beta_{0}>1.541$ and $\beta_{0}<\beta^{\prime} \leq 2$. Then for any constant $0<c<1$, there is a constant $d>0$ such that

$$
\left|\pi_{\beta_{1}}(I)\right| \geq c\left|\pi_{\beta}\left(I \cap S_{\beta}\right)\right|^{\frac{\log \beta_{1}}{\log \beta}}
$$

for any $\beta$ and $\beta_{1}$ with $\beta_{0}<\beta^{\prime}-d<\beta \leq \beta_{1}<\beta^{\prime}$, and any symbolic interval $I=[a, b] \subset S_{\beta_{1}}$. 
We postpone the proof of Lemma1 to Section 6. With the use of Lemma 1 we can now prove the following lemma.

Lemma 2. Let $\beta>1.541$ and let $w$ be a word of length $m$. Let $\left(\beta_{n}\right)_{n=1}^{\infty}$ be an increasing sequence in $(1.541, \beta)$ such that $S_{\beta_{n}}$ is of finite type and $\beta_{n} \rightarrow \beta$. For any $s<1$ there is an $N \in \mathbb{N}$, a $\delta>0$ and probability vectors $\bar{p}$ and $\bar{q}$ such that $\left|p_{w}-q_{w}\right|>\delta$ while

$$
\operatorname{dim}_{\mathrm{H}}\left(G_{\bar{p}}^{\beta_{n}, m}\right) \geq s \quad \text { and } \quad \operatorname{dim}_{\mathrm{H}}\left(G_{\bar{q}}^{\beta_{n}, m}\right) \geq s
$$

for all $n \geq N$.

Proof. Let $s<1$ be given and take $0<\sigma<1-s$. Then we can choose $N$ so large that

$$
\frac{\log \beta_{n}}{\log \beta}>1-\sigma \quad \text { for all } n \geq N
$$

Let $\bar{p}$ be a probability vector such that $G_{\bar{p}}^{\beta_{N}, m}$ has full dimension. Now, let $\delta>0$ be so small that there is a probability vector $\bar{q}$ with $\left|q_{w}-p_{w}\right|>\delta$ and $\operatorname{dim}_{\mathrm{H}}\left(G_{\bar{q}}^{\beta_{N}, m}\right) \geq s /(1-\sigma)$. This can be done according to Theorems 5 and 13 in [1], since $S_{\beta_{n}}$ is a subshift of finite type. There it is claimed that $\bar{p} \mapsto \operatorname{dim}_{\mathrm{H}}\left(G_{\bar{p}}^{\beta_{N}, m}\right)$ is real analytic (and obviously not constant), so it is clear that such a $q$ exists.

For $n \geq N$, and a set $A \subset S_{\beta_{N}}$, let $e_{n}(A)$ denote the embedding of $A$ into $S_{\beta_{n}}$. Then, by choosing $N$ sufficiently large, Lemma 1 implies that

$$
\operatorname{dim}_{\mathrm{H}}\left(e_{n}(A)\right) \geq \frac{\log \beta_{N}}{\log \beta_{n}} \operatorname{dim}_{\mathrm{H}}(A)>(1-\sigma) \operatorname{dim}_{\mathrm{H}}(A),
$$

so we get

$$
\operatorname{dim}_{M}\left(G_{\bar{q}}^{\beta_{n}, m}\right) \geq \operatorname{dim}_{\mathrm{H}}\left(e_{n}\left(G_{\bar{q}}^{\beta_{N}, m}\right)\right)>(1-\sigma) \operatorname{dim}_{\mathrm{H}}\left(G_{\bar{q}}^{\beta_{N}, m}\right)>s
$$

and

$$
\operatorname{dim}_{M}\left(G_{\bar{p}}^{\beta_{n}, m}\right) \geq \operatorname{dim}_{\mathrm{H}}\left(e_{n}\left(G_{\bar{p}}^{\beta_{N}, m}\right)\right)>(1-\sigma) \operatorname{dim}_{\mathrm{H}}\left(G_{\bar{p}}^{\beta_{N}, m}\right)=1-\sigma>s
$$

for all $n \geq N$.

Proof of Theorem 5. Let $\beta \in(1.541,2), s<1$ and $w \in S_{\beta}^{*}$ be a word of length $m$. Let $\left(\beta_{n}\right)_{n=1}^{\infty}$ be a sequence in $(1.541, \beta)$ such that $\beta_{n} \rightarrow \beta$ as $n \rightarrow \infty$ and $S_{\beta_{n}}$ is a subshift of finite type for each $n$. Indeed, that such a sequence exists was noted already in Section 4.1 .

According to Lemma 2 we can find $\delta>0$ and vectors $\bar{p}$ and $\bar{q}$ such that $\left|q_{w}-p_{w}\right|>4 \delta$ while $\operatorname{dim}_{\mathrm{H}}\left(G_{\bar{p}}^{\beta_{n}, m}\right)>s$ and $\operatorname{dim}_{\mathrm{H}}\left(G_{\bar{q}}^{\beta_{n}, m}\right)>s$ for all $n \geq N$ for some $N \in \mathbb{N}$.

Let $G_{k}^{1}$ be the set of generation $k$ cylinders in $S_{\beta}$ such that the frequency of each word $w$ of length $m$ is $\delta$-close to $p_{w}$. Let $G_{k}^{2}$ be the set of generation $k$ cylinders in $S_{\beta}$ such that the frequency of each word $w$ of length $m$ is $\delta$-close 
to $q_{w}$. It is no restriction to assume that there are words $u, v \neq 0^{m}$ such that $q_{w}>0$ and $p_{u}>0$. Indeed, if there were no such words, then we would not have $\operatorname{dim}_{\mathrm{H}}\left(G_{\bar{p}}^{\beta_{n}, m}\right)>s$ and $\operatorname{dim}_{\mathrm{H}}\left(G_{\bar{q}}^{\beta_{n}, m}\right)>s$.

By Proposition 8 the sets

$$
\pi_{\beta_{n}}\left(\bigcap_{M=1}^{\infty} \bigcup_{k=M}^{\infty} G_{k}^{1} \cap S_{\beta_{n}}\right) \text { and } \pi_{\beta_{n}}\left(\bigcap_{M=1}^{\infty} \bigcup_{k=M}^{\infty} G_{k}^{2} \cap S_{\beta_{n}}\right)
$$

are in the class $\mathcal{G}^{s}$ for all $n \geq N$, if $\delta$ is chosen small enough. Theorem 2 now implies that the $G_{\delta}$ sets

$$
\pi_{\beta}\left(\bigcap_{M=1}^{\infty} \bigcup_{k=M}^{\infty} G_{k}^{1}\right) \text { and } \pi_{\beta}\left(\bigcap_{M=1}^{\infty} \bigcup_{k=M}^{\infty} G_{k}^{2}\right)
$$

are in the class $\mathcal{G}^{s}$

To see that these sets are $G_{\delta}$ consider a point $x \in[0,1)$ that is on the boundary of $\pi_{\beta}\left(G_{k}^{1}\right)$. Then $\tau_{w}^{\beta}(x, n) /(n-m) \rightarrow 0$ as $n \rightarrow \infty$ for all words $w \neq 0^{m}$, making it impossible for $x$ to be in $\pi_{\beta}\left(G_{j}^{1}\right)$ for large $j$. Thus, we can just as well replace the sets $\pi_{\beta}\left(G_{k}^{1}\right)$ by their interiors.

\section{Proof of Theorem 1}

5.1. An auxiliary strategy. In this section we will prove the following lemma.

LEMma 3. The set

$$
\bigcup_{\beta^{\prime}<\beta} \pi_{\beta}\left(S_{\beta^{\prime}}\right)
$$

is $\alpha_{0}$-winning for any $\alpha_{0} \leq 1 / 16$ in the modified $(\alpha, \gamma)$-game.

In the proof we give a strategy for White to make sure that $\bigcap_{k=0}^{\infty} W_{k} \subset$ $\bigcup_{\beta^{\prime}<\beta} \pi_{\beta}\left(S_{\beta^{\prime}}\right)$. We call this strategy an auxiliary strategy, since it will be used in the next section to construct a winning strategy for the set $\pi_{\beta}(E)$ from Theorem 1 .

Proof of Lemma 3. Let $\alpha_{0}=1 / 16$. Before giving a winning strategy, let us introduce some notation. In the first step Black chooses $B_{0,0}$. Then White chooses $W_{0,0}$ and so on until Black and White have chosen $B_{0, k_{0}}$ and $W_{0, k_{0}}$. Then Black chooses $B_{1,0}$ and so on. This yields a sequence of natural numbers $\left(k_{j}\right)_{j=0}^{\infty}$ and sets $B_{j, k}$ and $W_{j, k}$ for any $j$ and $k \leq k_{j}$ such that

$$
B_{j-1, k_{j-1}} \supseteq W_{j-1, k_{j-1}} \supset B_{j, 0} \supseteq W_{j, 0} \supset \cdots \supset B_{j, k} \supseteq W_{j, k} \supset \cdots \supset B_{j, k_{j}} \cdots
$$

We now give the strategy for White to choose the sets $W_{j, k}$. Assume that Black has chosen $B_{j, 0}$. There is a smallest $n_{j}$ such that $B_{j, 0}$ contains a cylinder $C_{n_{j}}$ of generation $n_{j}$. (We identify $C_{n_{j}}$ with $\pi_{\beta}\left(s_{n_{j}}\right)$, where $s_{n_{j}}$ is 
a cylinder in $S_{\beta}$.) Let $D_{n_{j}}$ be the collection of all cylinders of generation $n_{j}$ which have non-empty intersection with $B_{j, 0}$.

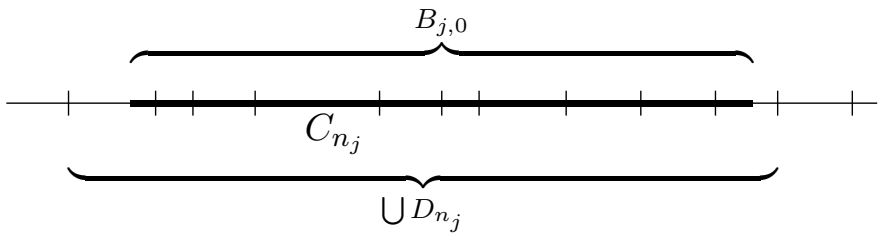

We then iterate:

White chooses $\alpha \in\left[\alpha_{0}, 1\right)$ and $W_{j, k} \subset B_{j, k}$ such that $W_{j, k}$ is a union of elements in $D_{n_{j}}$ (and the right endpoint, since $W_{j, k}$ is closed while the elements in $D_{n_{j}}$ are half-open). Black chooses $B_{j, k+1} \subset W_{j, k}$, until White cannot choose $W_{j, k}$ that is a union of elements in $D_{n_{j}}$.

We now have two cases:

CASE 1: $B_{j, k}$ has non-empty intersection with more than one element of $D_{n_{j}}$.

CASE 2: $B_{j, k}$ is contained in one element of $D_{n_{j}}$.

Observe that it can happen that the iteration above is not carried out, in which case we are in Case 1. We will treat each of the cases separately.

CASE 1: In this case $B_{j, k}$ has non-empty intersection with more than one element of $D_{n_{j}}$. We observe that if $D_{n_{j}}^{\prime} \subset D_{n_{j}}$ consists of the elements of $D_{n_{j}}$ that are subsets of $B_{j, k}$, then $\left|\bigcup D_{n_{j}}^{\prime}\right|<\alpha_{0} B_{j, k}$, since otherwise White can choose $W_{j, k}$ to be $\bigcup D_{n_{j}}^{\prime}$. Moreover there are at least one element and at most two elements of $D_{n_{j}} \backslash D_{n_{j}}^{\prime}$ that have non-empty intersection with $B_{j, k}$. Let $t_{0}$ be a cylinder such that $\left|B_{j, k} \cap t_{0}\right|$ is maximal. Then $\left|B_{j, k} \cap t_{0}\right|>$ $\frac{1-\alpha_{0}}{2}\left|B_{j, k}\right|>\frac{1}{4}\left|B_{j, k}\right|$. This means that by using a factor $1 / 4$ of the total $\alpha_{0}=1 / 16$, White has reduced the situation to Case 2 .

CASE 2: Let $s=\left[i_{0} \cdots i_{n_{j}+n-1}\right]$ be the smallest cylinder in which $B_{j, k}$ is contained. Let $k$ be the largest number such that $\left[i_{0} \cdots i_{n_{j}+n-1} 0^{k}\right]=$ $\left[i_{0} \cdots i_{n_{j}+n-1}\right]$. Let $s 0^{k} 0$ and $s 0^{k} 1$ denote the cylinders $\left[i_{0} \cdots i_{n_{j}+n-1} 0^{k} 0\right]$ and $\left[i_{0} \cdots i_{n_{j}+n-1} 0^{k} 1\right]$ respectively. We note that $s 0^{k} 0$ and $s 0^{k} 1$ are non-empty and $s=s 0^{k} 0 \cup s 0^{k} 1$.

If $\left|s 0^{k} 0 \cap B_{j, k}\right|>\frac{1}{4}\left|B_{j, k}\right|$ then we let $W_{j, k}=s 0^{k} 0 \cap B_{j, k}$. Otherwise we have $\left|s 0^{k} 1 \cap B_{j, k}\right|>\frac{3}{4}\left|B_{j, k}\right|$. Let $k^{\prime}$ be the largest number such that $\left[i_{0} \cdots i_{n_{j}+n-1} 0^{k} 10^{k^{\prime}}\right]=\left[i_{0} \cdots i_{n_{j}+n-1} 0^{k} 1\right]$. We denote $\left[i_{0} \cdots i_{n_{j}+n-1} 0^{k} 10^{k^{\prime}} 0\right]$ by $s 0^{k} 10^{k^{\prime}} 0$, and we get $\left|s 0^{k} 10^{k^{\prime}} 0 \cap B_{j, k}\right|>\frac{3}{4 \beta}\left|B_{j, k}\right|>\frac{1}{4}\left|B_{j, k}\right|$. Let $W_{j, k}=$ $s 0^{k} 10^{k^{\prime}} 0 \cap B_{j, k}$. 
This ends Case 2. Observe that in Case 2, White has only used at most a factor $1 / 4$ out of the total $\alpha_{0}=1 / 16$ that she is allowed to use, so in case we arrived at Case 2 from Case 1, White has used at most a factor 1/16 as allowed.

For $j \geq 1$, let $C_{j+1}$ be the cylinder $s 0^{k} 0$ or $s 0^{k} 10^{k^{\prime}} 0$ in which White was able to place $W_{j, k}$. We note that $\sigma^{k+1}\left(s 0^{k} 0\right)=\sigma^{k+1+k^{\prime}+1}\left(s 0^{k} 10^{k^{\prime}} 0\right)=S_{\beta}$. This means $C_{j}$ is just a scaled version of $[0,1)$. Let also $C_{0}=[0,1)$. We define $B_{j, k_{j}}=B_{j, k}$ and $W_{j, k_{j}}=W_{j, k}$, and let Black choose $B_{j+1,0}$.

We claim that there exists a number $N$ depending only on $\alpha_{0}, \gamma_{0}, \beta$ and $\left|B_{0,0}\right|$ such that the difference between the generations of the cylinders $C_{j+1}$ and $C_{j}$ is always smaller than $N$.

Note first that $\left|B_{j+1,0}\right| \geq\left|C_{j}\right| \min \left\{\left|B_{0,0}\right|, \alpha_{0} \gamma_{0}^{2}\right\}$. As already noted, each $C_{j}$ is a scaling of $[0,1)$ so for each $\alpha_{0}, \gamma_{0}, \beta$ and $\left|B_{0,0}\right|$ there are only finitely many possible $D_{n_{j}}$. Recall that $C_{j+1}$ was chosen as a subset of an element of $D_{n_{j}}$ depending on how Black chose $B_{j, k_{j}}$. If we let $E_{j+1}$ be the element of $D_{n_{j}}$ chosen, then we have $\left|C_{j+1}\right| \geq\left|W_{j, k_{j}}\right| \geq \alpha_{0}\left|B_{j, k_{j}}\right| \geq \alpha_{0} \gamma_{0}\left|E_{j+1}\right|$. Since there are finitely many possible $E_{j+1}$, there are finitely many possible $C_{j+1}$. Thus, for a fixed $j$, the difference in generation between $C_{j+1}$ and $C_{j}$ is bounded. Since changing $j$ only results in a scaling, this bound is independent of $j$, so there is indeed a number $N$ that bounds the difference between the generations of the cylinders $C_{j+1}$ and $C_{j}$ from above, for all $j$.

If White uses the strategy described above and if

$$
\left\{\pi_{\beta}\left(\left(i_{n}\right)_{n=0}^{\infty}\right)\right\}=\bigcap_{j=0}^{\infty} W_{j, 0}=\bigcap_{j=0}^{\infty} C_{j},
$$

then $\left(i_{n}\right)_{n=0}^{\infty}$ cannot contain sequences of $d(1, \beta)$ that are longer than $N$. Hence $\left(i_{n}\right)_{n=0}^{\infty} \in S_{\beta^{\prime}}$ if $\beta^{\prime}<\beta$ is such that $d_{n}\left(1, \beta^{\prime}\right)=d_{n}(1, \beta)$ for all $n \leq N$. This implies that the set

$$
\bigcup_{\beta^{\prime}<\beta} \pi_{\beta}\left(S_{\beta^{\prime}}\right)
$$

is $\left(\alpha_{0}, \gamma_{0}\right)$-winning for $\alpha_{0} \leq 1 / 16$ and any $\gamma_{0}$, i.e. it is $\alpha_{0}$-winning for $\alpha_{0} \leq 1 / 16$.

5.2. A winning strategy in the $(\alpha, \gamma)$-game. In this section we will use the auxiliary strategy to prove Theorem 1 . First we need some lemmata. Given an interval $I \subset[0,1)$ and $1<\beta^{\prime}<\beta<2$, let $I_{\left(\beta, \beta^{\prime}\right)} \subset[0,1)$ denote the interval $\pi_{\beta^{\prime}}\left(\pi_{\beta}^{-1}(I) \cap S_{\beta^{\prime}}\right)$.

Lemma 4. Let $\beta \in(1,2)$. For any $K \in(0,1)$ and $\varepsilon>0$, there is a $\beta_{0} \in$ $(1, \beta)$ such that for all intervals $I \subset[0,1)$ with $|I|>K$ the corresponding interval $I_{\left(\beta, \beta^{\prime}\right)}$ satisfies

$$
|I|(1-\varepsilon)<\left|I_{\left(\beta, \beta^{\prime}\right)}\right|<|I|(1+\varepsilon)
$$


and

$$
\left|I_{\left(\beta, \beta^{\prime}\right)}\right|(1-\varepsilon)<|I|<\left|I_{\left(\beta, \beta^{\prime}\right)}\right|(1+\varepsilon)
$$

for all $\beta^{\prime} \in\left[\beta_{0}, \beta\right)$.

Proof. This follows from the fact that the function

$$
[0,1) \ni x=\sum_{k=0}^{\infty} \frac{d_{k}\left(x, \beta^{\prime}\right)}{\left(\beta^{\prime}\right)^{k+1}} \mapsto \sum_{k=0}^{\infty} \frac{d_{k}\left(x, \beta^{\prime}\right)}{\beta^{k+1}},
$$

where $\beta^{\prime}<\beta$, converges uniformly to the identity as $\beta^{\prime} \rightarrow \beta$.

Lemma 5. Let $\beta \in(1,2)$. For any $K \in(0,1)$ and $\varepsilon>0$, there is a $\beta_{0} \in$ $(1, \beta)$ such that for all cylinders $\left[i_{0} \ldots i_{n-1} 0\right]$ in $S_{\beta}$ such that $\left[i_{0} \ldots i_{n-1} 0\right] \neq$ $\left[i_{0} \ldots i_{n-1}\right]$ and all intervals $I \subset\left[i_{0} \ldots i_{n-1} 0\right]$ with $|I|>K\left|\left[i_{0} \ldots i_{n-1} 0\right]\right|$ and such that all $1 / 4$-parts of I contain points from $\pi_{\beta}\left(S_{\beta_{0}}\right)$, the corresponding interval $I_{\left(\beta, \beta^{\prime}\right)}$ satisfies

$$
\frac{1}{2}|I|(1-\varepsilon)<\left|I_{\left(\beta, \beta^{\prime}\right)}\right| \frac{\beta^{\prime n+1}}{\beta^{n+1}}<|I|(1+\varepsilon)
$$

and

$$
\left|I_{\left(\beta, \beta^{\prime}\right)}\right|(1-\varepsilon)<|I| \frac{\beta^{n+1}}{\beta^{\prime n+1}}<2\left|I_{\left(\beta, \beta^{\prime}\right)}\right|(1+\varepsilon)
$$

for all $\beta^{\prime} \in\left[\beta_{0}, \beta\right)$.

Proof. Fix $K$ and let $I$ satisfy the assumptions. We have

$$
I=\left(\sum_{i=0}^{\infty} \frac{a_{i}}{\beta^{i+1}}, \sum_{i=0}^{\infty} \frac{b_{i}}{\beta^{i+1}}\right),
$$

where $\left(a_{i}\right)_{i=0}^{\infty},\left(b_{i}\right)_{i=0}^{\infty} \in S_{\beta}$. Let $I^{\prime}$ be the largest interval

$$
I^{\prime}=\left(\sum_{i=0}^{\infty} \frac{a_{i}^{\prime}}{\beta^{i+1}}, \sum_{i=0}^{\infty} \frac{b_{i}^{\prime}}{\beta^{i+1}}\right) \subset I
$$

such that $\left(a_{i}^{\prime}\right)_{i=0}^{\infty},\left(b_{i}^{\prime}\right)_{i=0}^{\infty} \in S_{\beta^{\prime}}$. Note that this implies $I_{\left(\beta, \beta^{\prime}\right)}^{\prime}=I_{\left(\beta, \beta^{\prime}\right)}$. By the assumptions on $I$ we also have $\left|I^{\prime}\right|>\frac{1}{2}|I|$. Moreover,

$$
\begin{aligned}
\left|I^{\prime}\right| & =\left|\sum_{i=0}^{\infty} \frac{a_{i}^{\prime}-b_{i}^{\prime}}{\beta^{i+1}}\right|=\left|\sum_{i=n+1}^{\infty} \frac{a_{i}^{\prime}-b_{i}^{\prime}}{\beta^{i+1}}\right| \\
& =\beta^{-(n+1)}\left|\sum_{i=0}^{\infty} \frac{a_{i+n+1}^{\prime}-b_{i+n+1}^{\prime}}{\beta^{i+1}}\right|=\beta^{-(n+1)}\left|J^{\prime}\right|,
\end{aligned}
$$

where

$$
J^{\prime}=\left(\sum_{i=0}^{\infty} \frac{a_{i+n+1}^{\prime}}{\beta^{i+1}}, \sum_{i=0}^{\infty} \frac{b_{i+n+1}^{\prime}}{\beta^{i+1}}\right) \subset[0,1)
$$

and $\left|J^{\prime}\right|>K$. 
By Lemma 4 we can choose $\beta_{0}$ such that

$$
\left|J^{\prime}\right|(1-\varepsilon)<\left|J_{\left(\beta, \beta^{\prime}\right)}^{\prime}\right|<\left|J^{\prime}\right|(1+\varepsilon)
$$

and

$$
\left|J_{\left(\beta, \beta^{\prime}\right)}^{\prime}\right|(1-\varepsilon)<\left|J^{\prime}\right|<\left|J_{\left(\beta, \beta^{\prime}\right)}^{\prime}\right|(1+\varepsilon)
$$

for all $\beta^{\prime} \in\left[\beta_{0}, \beta\right)$. Since $\left(a_{i}^{\prime}\right)_{i=0}^{\infty},\left(b_{i}^{\prime}\right)_{i=0}^{\infty} \in S_{\beta^{\prime}}$, we have

$$
I_{\left(\beta, \beta^{\prime}\right)}^{\prime}=\left(\sum_{i=0}^{\infty} \frac{a_{i}^{\prime}}{\left(\beta^{\prime}\right)^{i+1}}, \sum_{i=0}^{\infty} \frac{b_{i}^{\prime}}{\left(\beta^{\prime}\right)^{i+1}}\right)
$$

and

$$
J_{\left(\beta, \beta^{\prime}\right)}^{\prime}=\left(\sum_{i=0}^{\infty} \frac{a_{i+n+1}^{\prime}}{\left(\beta^{\prime}\right)^{i+1}}, \sum_{i=0}^{\infty} \frac{b_{i+n+1}^{\prime}}{\left(\beta^{\prime}\right)^{i+1}}\right) .
$$

It follows that

$$
\begin{aligned}
\left|I_{\left(\beta, \beta^{\prime}\right)}^{\prime}\right| & =\left|\sum_{i=0}^{\infty} \frac{a_{i}^{\prime}-b_{i}^{\prime}}{\left(\beta^{\prime}\right)^{i+1}}\right|=\left|\sum_{i=n+1}^{\infty} \frac{a_{i}^{\prime}-b_{i}^{\prime}}{\left(\beta^{\prime}\right)^{i+1}}\right| \\
& =\beta^{\prime-(n+1)}\left|\sum_{i=0}^{\infty} \frac{a_{i+n+1}^{\prime}-b_{i+n+1}^{\prime}}{\left(\beta^{\prime}\right)^{i+1}}\right|=\beta^{-(n+1)}\left|J_{\left(\beta, \beta^{\prime}\right)}^{\prime}\right| .
\end{aligned}
$$

This implies

$$
\left|I^{\prime}\right|(1-\varepsilon)<\left|I_{\left(\beta, \beta^{\prime}\right)}^{\prime}\right| \frac{\beta^{\prime n+1}}{\beta^{n+1}}<\left|I^{\prime}\right|(1+\varepsilon)
$$

and

$$
\left|I_{\left(\beta, \beta^{\prime}\right)}^{\prime}\right|(1-\varepsilon)<\left|I^{\prime}\right| \frac{\beta^{n+1}}{\beta^{\prime n+1}}<\left|I_{\left(\beta, \beta^{\prime}\right)}^{\prime}\right|(1+\varepsilon) .
$$

Using $\left|I^{\prime}\right|<|I|<2\left|I^{\prime}\right|$ and $\left|I_{\left(\beta, \beta^{\prime}\right)}^{\prime}\right|=\left|I_{\left(\beta, \beta^{\prime}\right)}\right|$ we get

$$
\frac{1}{2}|I|(1-\varepsilon)<\left|I_{\left(\beta, \beta^{\prime}\right)}\right| \frac{\beta^{\prime n+1}}{\beta^{n+1}}<|I|(1+\varepsilon)
$$

and

$$
\left|I_{\left(\beta, \beta^{\prime}\right)}\right|(1-\varepsilon)<|I| \frac{\beta^{n+1}}{\beta^{\prime n+1}}<2\left|I_{\left(\beta, \beta^{\prime}\right)}\right|(1+\varepsilon) .
$$

We are now ready to prove Theorem 1 .

Proof of Theorem 1. We will show that there is a strategy for White such that for each $\gamma_{0}>0,\left|B_{0}\right|>0$ and $\beta \in(1,2)$, there is a $\beta^{*}<\beta$ such that White can make sure that in the $\left(\alpha_{0}, \gamma_{0}\right)$-game we get $\bigcap_{i=1}^{\infty} W_{i} \subset \pi_{\beta}\left(S_{\beta^{*}} \cap E\right)$ if $\alpha_{0} \leq \min \{1 / 16, \alpha / 4\}$. First of all we note that it is sufficient to prove this for small $\gamma_{0}$, for example $\gamma_{0} \leq 1 / 4$. Indeed, a winning strategy for White from the $\left(\alpha_{0}, \gamma_{0}\right)$-game works just as well in any $\left(\alpha_{0}, \gamma_{1}\right)$-game where $\gamma_{1}>\gamma_{0}$.

The game begins when Black chooses $B_{0}$. The first task for White is to make sure that the game stays in $\pi_{\beta}\left(S_{\beta^{*}}\right)$. By Lemma 3 we know that there 
is a strategy for White that enables her to do this for $\alpha \leq 1 / 16$. We let White play $W_{i}$ according to this strategy for all even turns $i$. That she does not play each time can be interpreted as that $\gamma_{0}$ is replaced by $\alpha_{0} \gamma_{0}^{2}$, which is no problem for White since she can handle any $\gamma_{0}>0$. So, by Lemma 3 . White can make sure that $\bigcap_{i=1}^{\infty} W_{i} \subset \pi_{\beta}\left(S_{\beta^{*}}\right)$ for some $\beta^{*} \in(1, \beta)$.

We know from the proof of Lemma 3 that the strategy White uses at the even turns ensures that after playing the game a finite number of turns, we have $W_{i_{1}} \subset C_{i_{1}}$, where $C_{i_{1}}=\pi_{\beta}\left(s_{i_{1}}\right)$ is a generation $i_{1}$ cylinder in $S_{\beta}$ which is a scaling of $[0,1)$ such that $s_{i_{1}}$ is non-empty as a cylinder in $S_{\beta^{*}}$, and $\left|W_{i_{1}}\right| \geq K\left|C_{i_{1}}\right|$ for some $K>0$. After this is done, White starts over at the next even turn and sooner or later achieves $W_{i_{2}} \subset C_{i_{2}}$, where $C_{i_{2}}=\pi_{\beta}\left(s_{2}\right)$ again is a scaling of $[0,1)$ such that $s_{2}$ is non-empty as a cylinder in $S_{\beta^{*}}$ and $\left|W_{i_{2}}\right| \geq K\left|C_{i_{2}}\right|$. It follows from the proof of Lemma 3 that $i_{2}-i_{1}$ is bounded. This implies that $\left|W_{i_{2}}\right| \geq L\left|W_{i_{1}}\right|$, where $L>0$ depends only on $\alpha_{0}, \beta, \gamma_{0}$ and $\left|B_{0}\right|$.

Consider now the odd turn for White that takes place after White played $W_{i_{k}} \subset C_{i_{k}}$ and Black played $B_{i_{1}+1}$. Obviously we have $\left|W_{i_{k}+1}\right| \leq\left|W_{i_{k}}\right|$. Compare this with the next time White is in the same situation. Then she has to play $W_{i_{k+1}+1} \subset B_{i_{k+1}+1}$. But $\left|B_{i_{k+1}+1}\right| \geq \gamma_{0}\left|W_{i_{k+1}}\right| \geq \gamma_{0} L\left|W_{i_{k}}\right|$ so if White plays only at these special situations, then it appears to White as if her opponent has played with $\gamma_{0} L$ instead of just $\gamma_{0}$.

We will now use Lemma 5. White is given an interval $B_{i_{k}+1}$ which constitutes at least a fraction $\alpha_{0} \gamma_{0}^{2}$ of a cylinder $C_{i_{k}}=\pi_{\beta}\left(s_{i_{k}}\right)$ that is a scaling of $[0,1)$ and such that $s_{i_{k}}$ is non-empty as a cylinder in $S_{\beta^{*}}$. Regardless of how White plays, the interval $W_{i_{k}+1}$ constitutes at least a fraction $\alpha_{0}^{2} \gamma_{0}^{2}$ of the same cylinder. Since we have already made sure that $\bigcap_{i=1}^{\infty} W_{i} \subset \pi_{\beta}\left(S_{\beta^{*}}\right)$, it is clear that every $1 / 4$ part of $B_{i_{k}+1}$ (as well as $B_{i_{k+1}+1}$ ) contains points from $\pi_{\beta}\left(S_{\beta^{*}}\right)$. Letting $A^{*}$ denote $A_{\left(\beta, \beta^{*}\right)}$ for a set $A$, Lemma 5 then implies

$$
\frac{\left|B_{i_{k+1}+1}^{*}\right|}{\left|W_{i_{k}+1}^{*}\right|}>\frac{1-\varepsilon}{2(1+\varepsilon)} \frac{\left|B_{i_{k+1}+1}\right|}{\left|W_{i_{k}+1}\right|} \geq \gamma_{0} L \frac{1-\varepsilon}{2(1+\varepsilon)} .
$$

Thus, if we consider the game in $S_{\beta^{*}}$, it is as if Black played with $2^{-1} \gamma_{0} L \frac{1-\varepsilon}{1+\varepsilon}$ instead of $\gamma_{0}$. But this is no problem since the set $E$ is winning for all $\gamma_{0}>0$ as long as $\alpha_{0} \leq \alpha$. We want to allow White to use her strategy from $S_{\beta^{*}}$ to choose a set $W_{i_{k}+1}^{*}$ such that $\left|W_{i_{k}+1}^{*}\right| \geq \alpha\left|B_{i_{k}+1}^{*}\right|$. In $S_{\beta}$, this would give us a set $W_{i_{k}+1} \subset B_{i_{k}+1}$. Again by Lemma 5 we get

$$
\frac{\left|W_{i_{k}+1}\right|}{\left|B_{i_{k}+1}\right|}>\frac{1-\varepsilon}{2(1+\varepsilon)} \frac{\left|W_{i_{k}+1}^{*}\right|}{\left|B_{i_{k}+1}^{*}\right|} \geq \frac{\alpha}{2} \frac{1-\varepsilon}{1+\varepsilon} .
$$

Thus, if we use $\alpha_{0} \leq \min \{1 / 16, \alpha / 4\}$, we can choose $\beta_{0}$ such that White gets $\alpha$ to play with in $S_{\beta^{*}}$. So, in $S_{\beta^{*}}$ White plays an $\left(\alpha_{0}, \gamma_{0}\right)$-game with $\alpha_{0} \leq \alpha$ 
and $\gamma_{0}>0$. By assumption, there is a strategy White can use to make sure that $\bigcap_{k=1}^{\infty} W_{i_{k}+1} \subset \pi_{\beta}\left(E \cap S_{\beta}^{*}\right)$.

We can do this for any $\gamma_{0}>0$ and any $\left|B_{0}\right|$. Thus the set

$$
\pi_{\beta}(E) \supset \bigcup_{\beta^{\prime}<\beta} \pi_{\beta}\left(E \cap S_{\beta}^{\prime}\right)
$$

is $\alpha_{0}$-winning in $[0,1]$ for all $\alpha_{0} \leq \min \{1 / 16, \alpha / 4\}$.

6. Proof of Theorem 2. The proof of Theorem 2 will rely on the following lemma by Solomyak. We refer to [13 for a proof.

Lemma 6. Let $x_{0}<0.649$. There is a constant $\delta>0$ such that if $x \in$ $\left[0, x_{0}\right]$ then the implication

$$
g(x) \leq \delta \Rightarrow g^{\prime}(x)<-\delta
$$

holds for any function $g(x)$ of the form

$$
g(x)=1+\sum_{k=1}^{\infty} a_{k} x^{k}, \quad a_{k} \in\{-1,0,1\} .
$$

COROLlary 4. Let

$$
g(\beta)=1+\sum_{k=1}^{\infty} \frac{a_{k}}{\beta^{k}}, \quad a_{k} \in\{-1,0,1\},
$$

and $\beta_{0}>1.541$. Then there is a constant $\delta$ such that if $\beta>\beta_{0}$ then

$$
g(\beta)<\delta \Rightarrow g^{\prime}(\beta)>\delta \beta^{-2} .
$$

Proof. Let $x_{0}=\beta_{0}^{-1}$ and take $\delta$ according to Lemma 6. Let $i(x)=x^{-1}$ and

$$
h(x)=1+\sum_{k=1}^{\infty} a_{n+k} x^{k} .
$$

Then $g(\beta)=h \circ i(\beta)$ and $g^{\prime}(\beta)=-h^{\prime}(i(\beta)) \beta^{-2}$.

If $g(\beta)<\delta$ then $h(i(\beta))<\delta$ and by Lemma 6 we have $h^{\prime}(i(\beta))<-\delta$ and so $g^{\prime}(\beta)>\delta \beta^{-2}$.

We are now ready to prove Lemma 1, which was already stated in Section 4.2. For convenience we restate it here.

Lemma 1. Let $\beta_{0}>1.541$ and $\beta_{0}<\beta^{\prime} \leq 2$. Then for any constant $0<c<1$, there is a constant $d>0$ such that

$$
\left|\pi_{\beta_{1}}(I)\right| \geq c\left|\pi_{\beta}\left(I \cap S_{\beta}\right)\right|^{\frac{\log \beta_{1}}{\log \beta}},
$$

for any $\beta$ and $\beta_{1}$ with $\beta_{0}<\beta^{\prime}-d<\beta \leq \beta_{1}<\beta^{\prime}$, and any symbolic interval $I=[a, b] \subset S_{\beta_{1}}$. 
Proof. Fix $\beta_{0}, \beta_{1}$ and pick $0<\delta<1$ according to Corollary 4. It is sufficient to prove the lemma in the case when $a, b \in S_{\beta}$ and $a<b$.

Let

$$
f(t)=\left(\sum_{k=0}^{\infty} \frac{b_{k}-a_{k}}{t^{k+1}}\right)^{\frac{\log \beta_{1}}{\log t}} .
$$

Then $f\left(\beta_{1}\right)=\left|\pi_{\beta_{1}}(I)\right|$ and $f(\beta)=\left|\pi_{\beta}\left(I \cap S_{\beta}\right)\right|^{\frac{\log \beta_{1}}{\log \beta}}$. We will show that $f\left(\beta_{1}\right)>c f(\beta)$.

If $n-1$ is the smallest number such that $a_{n-1} \neq b_{n-1}$, then $a_{n-1}=0$, $b_{n-1}=1$ and

$$
f(t)=\beta_{1}^{-n}\left(1+\sum_{k=0}^{\infty} \frac{b_{k+n}-a_{k+n}}{t^{k+1}}\right)^{\frac{\log \beta_{1}}{\log t}} .
$$

Let

$$
g(t)=1+\sum_{k=0}^{\infty} \frac{b_{k+n}-a_{k+n}}{t^{k+1}} .
$$

Then $g(\beta)>0$. We have $f(\beta)=\beta_{1}^{-n} g(\beta)^{\frac{\log \beta_{1}}{\log \beta}}$. This implies that

$$
f^{\prime}(\beta)=\beta_{1}^{-n} g(\beta)^{\frac{\log \beta_{1}}{\log \beta}}\left(-\frac{\log \beta_{1}}{(\log \beta)^{2}} \frac{1}{\beta} \log g(\beta)+\frac{\log \beta_{1}}{\log \beta} \frac{g^{\prime}(\beta)}{g(\beta)}\right) .
$$

By (6.1) and Corollary 4, we observe $f^{\prime}(\beta)>0$ if $g(\beta)<\delta<1$, since then both terms in the parentheses are positive. Hence if $g(\beta)<\delta$ then $f(\beta)<f\left(\beta_{1}\right)$.

Otherwise, if $g(\beta) \geq \delta$, we first observe that

$$
\left|g^{\prime}(\beta)\right| \leq \sum_{k=1}^{\infty} \frac{k}{\beta^{k+1}}=\frac{1}{(\beta-1)^{2}}<\frac{1}{\left(\beta_{0}-1\right)^{2}} .
$$

Then by 6.1) we get

$$
\begin{aligned}
\left|f^{\prime}(\beta)\right| & \leq \beta_{1}^{-n} g(\beta)^{\frac{\log \beta_{1}}{\log \beta}}\left(-\frac{\log \beta_{1}}{(\log \beta)^{2}} \frac{1}{\beta} \log \delta+\frac{\log \beta_{1}}{\log \beta} \frac{\left(\beta_{0}-1\right)^{-2}}{\delta}\right) \\
& \leq f(\beta)\left(-\frac{\log \beta_{1}}{\left(\log \beta_{0}\right)^{2}} \frac{1}{\beta_{0}} \log \delta+\frac{\log \beta_{1}}{\log \beta_{0}} \frac{1}{\delta\left(\beta_{0}-1\right)^{2}}\right)=C f(\beta),
\end{aligned}
$$

where $C$ only depends on $\beta_{0}$ and $\beta^{\prime}$. Hence $f\left(\beta_{1}\right)>c f(\beta)$ for all $\beta \leq \beta_{1}<\beta^{\prime}$ provided that $\beta^{\prime}-\beta<(\log c) / C$. Take $0<d<(\log c) / C$.

We are now ready to prove Theorem 2 .

Proof of Theorem 2. Given an interval $J \subset[0,1)$, let $\tilde{J} \subset[0,1)$ denote the interval we get if we take $\pi_{\beta_{n}}\left(\pi_{\beta}^{-1}(J) \cap S_{\beta_{n}}\right)$. 
Let $I$ be any interval in $[0,1)$ and let $\epsilon>0$ be so small that $|I|^{\epsilon} \geq 1 / 2$. Use Lemma 1 to find $n_{0}$ such that

$$
|J| \geq \frac{1}{2}|\tilde{J}|^{\frac{\log \beta}{\log \beta_{n}}}
$$

for all intervals $J \subset[0,1)$ and all $n \geq n_{0}$. Choose $n_{1}>n_{0}$ so large that $(s-\epsilon) \frac{\log \beta}{\log \beta_{n}}<s$ for all $n \geq n_{1}$. Choose $n_{2}>n_{1}$ so large that $|\tilde{I}|^{s}>\frac{1}{2}|I|^{s}$ for all $n \geq n_{2}$.

Let $\left(U_{i}\right)$ be any cover of $F \cap I$ with $U_{i} \subset[0,1)$ for all $i$. Then $\left(\tilde{U}_{i}\right)$ is a cover of

$$
\pi_{\beta_{n}}\left(\pi_{\beta}^{-1}(F \cap I) \cap S_{\beta_{n}}\right) \supset \pi_{\beta_{n}}\left(E \cap S_{\beta_{n}}\right) \cap \tilde{I} .
$$

Since $\pi_{\beta_{n}}\left(E \cap S_{\beta_{n}}\right)$ is in $\mathcal{G}^{s}$ for all $n$, we get

$$
\begin{aligned}
\sum\left|U_{i}\right|^{s-\epsilon} & >\sum \frac{1}{2}\left|\tilde{U}_{i}\right|^{(s-\epsilon) \frac{\log \beta}{\log \beta_{n}}}>\sum \frac{1}{2}\left|\tilde{U}_{i}\right|^{s} \\
& \geq \frac{1}{2} \mathcal{M}_{\infty}^{s}\left(\pi_{\beta_{n}}\left(E \cap S_{\beta_{n}}\right) \cap \tilde{I}\right) \geq \frac{1}{2}|\tilde{I}|^{s}>\frac{1}{4}|I|^{s}>\frac{1}{8}|I|^{s-\epsilon}
\end{aligned}
$$

for all $n \geq n_{2}$. Since the cover $\left(U_{i}\right)$ was arbitrary we get

$$
\mathcal{M}_{\infty}^{s-\epsilon}(F \cap I) \geq \frac{1}{8}|I|^{s-\epsilon} .
$$

Since the interval $I \subset[0,1)$ was arbitrary and $F$ is a $G_{\delta}$ set, Proposition 5 implies that $F$ is in the class $\mathcal{G}^{s-\epsilon}$. But we can do this for arbitrarily small $\epsilon>0$, so $F$ is in the class $\mathcal{G}^{t}$ for all $t<s$. Thus, by Proposition 7, $F$ is in the class $\mathcal{G}^{s}$.

\section{References}

[1] L. Barreira, B. Saussol, and J. Schmeling, Higher-dimensional multifractal analysis, J. Math. Pures Appl. (9) 81 (2002), 67-91.

[2] L. Barreira and J. Schmeling, Sets of "non-typical" points have full topological entropy and full Hausdorff dimension, Israel J. Math. 116 (2000), 29-70.

[3] D. Färm, Simultaneously non-dense orbits under different expanding maps, Monatsh. Math., to appear.

[4] - Simultaneously non-convergent frequencies of words in different expansions, Dynam. Systems, to appear.

[5] K. Falconer, Classes of sets with large intersection, Mathematika 32 (1985), 191-205.

[6] - Sets with large intersection properties, J. London Math. Soc. (2) 49 (1994), 267-280.

[7] —, Fractal Geometry. Mathematical Foundations and Applications, Wiley, Chichester, 1990.

[8] J. Nilsson, On numbers badly approximable by dyadic rationals, Israel J. Math. 171 (2009), 93-110.

[9] - On numbers badly approximable via the $\beta$-shift, preprint.

[10] W. Parry, On the $\beta$-expansion of real numbers, Acta Math. Acad. Sci. Hungar. 11 (1960), 401-416. 
[11] T. Persson and J. Schmeling, Dyadic Diophantine approximation and Katok's horseshoe approximation, Acta Arith. 132 (2008), 205-230.

[12] W. Schmidt, On badly approximable numbers and certain games, Trans. Amer. Math. Soc. 123 (1966), 178-199.

[13] B. Solomyak, On the random series $\sum \pm \lambda^{n}$ (an Erdôs problem), Ann. of Math. (2) 142 (1995), 611-625.

David Färm

Institute of Mathematics

Polish Academy of Sciences

Śniadeckich 8

00-956 Warszawa, Poland

and

Centre for Mathematical Sciences

Lund University

Box 118

SE-22100 Lund, Sweden

E-mail: D.Farm@impan.pl
Tomas Persson

Institute of Mathematics

Polish Academy of Sciences

Śniadeckich 8

00-956 Warszawa, Poland

E-mail: T.Persson@impan.pl

Jörg Schmeling

Centre for Mathematical Sciences

Lund University

Box 118

SE-22100 Lund, Sweden

E-mail: joerg@maths.lth.se

Received 29 January 2009;

in revised form 10 March 2010 